\title{
Expression of PSMA in Thyroid Follicular Neoplasms: Utility for Differentiating Between Benign from Malignant Lesions
}

\author{
Hiva Saffar*, Marzieh Kiany, Seyed Mohammad Tavangar and Elham Mirzaian \\ Department of Pathology, Shariati Hospital, Tehran University of Medical Sciences, Tehran, Iran
}

\begin{abstract}
Background: Prostate-Specific Membrane Antigen (PSMA) has been known as a tumor associated neovasculature marker in some solid malignant tumors. Targeting of PSMA by cytotoxic- conjugated antibody can represent powerful tool for vascular targeted therapy. In thyroid, it appears that angiogenesis factors are involved in neoplastic growth and aggressiveness of tumors.

This study was conducted to evaluate the expression of PSMA as an angiogenesis factor in neovasculature of thyroid follicular neoplasms by immunohistochemistry to determine its usefulness for distinguishing between adenoma and carcinoma.

Methods: Paraffin Blocks of formalin fixed samples representing 48 cases of Follicular Thyroid Adenoma (FTA) and 15 cases of Follicular Thyroid Carcinoma (FTC) were evaluated.

Results: Among 15 cases of FTC, 11 cases revealed both capsular and vascular invasion. In one case only capsular and in two cases only vascular invasions were observed. One case also revealed suspicious vascular invasion with further bone metastasis.

The intensity of PSMA staining in vascular structures of carcinomas were significantly higher in comparison with thyroid follicular adenomas $(\mathrm{p}=0.028)$. However, we observed no significant difference in the extent of PSMA expression in adenoma and carcinoma groups $(\mathrm{p}=0.239)$. Thyroid follicular cells were negative for PSMA in adenoma, carcinoma and in non-neoplastic area.

Conclusion: According to our findings, significant higher intensity of PSMA expression in FTC could be useful in differentiating between FTA and FTC. However, more importantly, expression in neovasculature of differentiated thyroid tumors can represent a potential utility for PSMA-targeted radionuclide therapy in advanced differentiated thyroid cancers.
\end{abstract}

Keywords: Angiogenesis, Prostate-Specific Membrane Antigen, Thyroid Follicular Adenoma, Thyroid Follicular Carcinoma

\section{Introduction}

Thyroid cancers are the most common endocrine malignancies that have increased recently. ${ }^{[1]}$ The number of cases with thyroid nodule requiring further evaluation even is higher, probably due to use of more sensitive detection techniques. ${ }^{[2]}$ Thyroid lesions with follicular growth pattern are composed of a wide range including benign adenomatous nodule, Follicular Thyroid Adenoma (FTA), Follicular Thyroid carcinoma (FTC) and Follicular variant of Papillary Thyroid Carcinoma (FVPTC). ${ }^{[3-5]}$

FTA, FTC and FVPTC are well encapsulated lesions sharing many imaging, cytologic and histologic features ${ }^{[5-8]}$.

Difficulties are also not uncommon while reporting these follicular pattern neoplasms based on histomorphological findings. ${ }^{[9]}$ Thus, diagnosis of a solitary encapsulated nodule with follicular histologic pattern is sometimes problematic for pathologists. ${ }^{[5,10]}$

One of the most challenging tasks in pathologic evaluation of thyroid follicular pattern lesion is distinction between FTC and FTA as a common benign thyroid nodule. ${ }^{[4]}$
Diagnosis of carcinoma is based on the presence of capsular, vascular or extra thyroid tissue invasion or nodal or distant metastasis. ${ }^{[5,8,10-12]}$

Different tools such as IHC and molecular profiling have been used to differentiate between benign and malignant follicular neoplasms or differentiating malignant tumor subtypes, $[4,9$, ${ }^{13]}$ including some angiogenesis factors. ${ }^{[9]}$ Thyroid is rich in vascular network ${ }^{[14]}$ and shows increased vascularity in both non neoplastic and malignant conditions. ${ }^{[9]}$

On the other hand, a system of nutrient vessels is an essential component of tumor growth. ${ }^{[15]}$ For example, expression of VEGF, a hypoxia induced angiogenic factor, concomitant with hyper vascularity have been induced more strongly in differentiated malignant thyroid tumors than benign tumors. ${ }^{[16]}$ Micro-vessel Density (MVD) as an indicator of angiogenesis is shown to be correlated with disease free survival in PTC and intra thyroid tumor spread in FTC. $\left.{ }^{9,}, 14,17\right]$

These data suggest that angiogenesis factors are involved in neoplastic growth and aggressiveness of thyroid tumors. 
[14] Thus, monitoring the process of angiogenesis may represent an early tool for diagnosis of malignancy. ${ }^{[18]}$

Prostate Specific Membrane Antigen (PSMA) is type II integral membrane glycoprotein initially expressed in prostate cancer cells but then it had been discovered that it also is expressed in neovasculature of the various solid tumors, including some carcinomas, neuroendocrine tumors, sarcomas and melanomas ${ }^{[15,19-21]}$, but neither normal endothelium ${ }^{[15]}$ nor endothelial cells of benign tissue are positive. ${ }^{[19]}$ Recently, it has been suggested that PSMA expression has been evident in some thyroid neoplasms using $\left[{ }^{68} \mathrm{Ga}\right]$ PSMA-HBED-ccPET/CT. ${ }^{[22-26]}$

This study is conducted to evaluate the expression of PSMA by Immunohistochemistry in neovasculature of thyroid follicular neoplasms to determine its usefulness for distinguishing between adenoma and carcinoma. Also, to evaluate the potential capability of radio labeled Anti PSMA antibody as complementary target therapy in advanced thyroid malignancies.

\section{Materials \& Methods}

Paraffin Blocks of formalin fixed samples representing 48 cases of FTA and 15 cases of FTC were retrieved from archive of pathology department . The questionable cases were excluded. Blocks with minimal necrosis or hemorrhage and representative amount of tumor tissue were selected for IHC study. IHC was performed using monoclonal liquid NCL-L-PSMA mouse antibody (Clone 1D6, Novocastra) according to the manufacturers' protocol. Prostate tissue was considered as positive control. Also we performed CD31 staining (JC/70A,Biogenex) to confirm the localization of neovasculature.

The stained sections were assessed for the extent and intensity of endothelial cell staining in tumor micro-vessels and scored semi-quantitatively.(table-1)
After data collection, all quantitative and qualitative data were analyzed using statistical package for Social Sciences (SPSS) version 19. P-value less than 0.05 was considered significant.

\section{Results}

Totally, 63 cases of thyroid follicular neoplasms were evaluated including 48 cases of follicular thyroid adenoma and 15 cases of follicular thyroid carcinomas. Among FTCs, 11 cases revealed both capsular and vascular invasion. In one case only capsular and in two cases only vascular invasions were observed. One case also revealed suspicious vascular invasion with further bone metastasis.

Female: male ratio in adenoma and carcinoma groups were $37: 11$ and 9:6, respectively. The average age of the patients in adenoma and carcinoma groups was $35.8 \pm 10.1$ and $43.8 \pm 13.4$ years, respectively. Thus, the patients with carcinoma were significantly older than adenoma group. $(p=0.029)$. Regarding to the size of the tumor, we did not find any significant relationship. $(3.3 \pm 1.7 \mathrm{~cm}$ in adenoma versus $3.7 \pm 2.4 \mathrm{~cm}$ in carcinoma; $\mathrm{p}=0.597$ ).

We evaluated the extent and intensity of PSMA staining in vascular endothelial cells and scored semi-quantitatively (Table-1). The results for PSMA IHC staining are summarized in table 2 and 3. The intensity of PSMA staining was significantly higher in carcinoma rather than adenoma cases $(\mathrm{p}=0.028)($ Table-2). However, we did not observe any significant difference in the extent of PSMA expression in vascular structures of thyroid adenoma and carcinoma groups $(\mathrm{p}=0.239)($ Table-3). Thyroid follicular cells in adenoma, carcinoma also non neoplastic area were negative for PSMA.

\section{Discussion}

Reliable determination of thyroid tumor pathology could reduce the cost of management of thyroid nodules by eliminating many of the diagnostic thyroidectomies

Table 1: Scoring system for the Extent and Intensity of PSMA in IHC staining

\begin{tabular}{|l|c|c|c|}
\hline $\begin{array}{l}\text { Percentage of stained } \\
\text { endothelial cells(\%) }\end{array}$ & Interpretation & Intensity & Interpretation \\
\hline $0-9$ & Negative & No reaction & 0 \\
\hline $10-39$ & Minimal & Faint reaction visible only at high power & $1+$ \\
\hline $40-69$ & Moderate & Moderate intensity at low power & $2+$ \\
\hline$\geq 70$ & Diffuse & Strong reaction easily visible at low power & $3+$ \\
\hline
\end{tabular}

Table 2: The intensity of PSMA staining by immunohistochemistry in FTA and FTC

\begin{tabular}{|l|c|c|c|c|c|}
\hline Intensity Score, Histologic Dx & $\mathbf{0}$ & $\mathbf{1}$ & $\mathbf{2}$ & $\mathbf{3}$ & Total \\
\hline Follicular Thyroid Adenoma & $39(81.25 \%)$ & $6(12.50 \%)$ & $1(2.08 \%)$ & $2(4.17 \%)$ & $\mathbf{4 8}$ \\
\hline Follicular Thyroid Carcinoma & $10(66.67 \%)$ & $1(6.67 \%)$ & $4(26.66 \%)$ & $0(0.00 \%)$ & $\mathbf{1 5}$ \\
\hline
\end{tabular}


Table 3: The extent of PSMA staining by immunohistochemistry in FTA and FTC

\begin{tabular}{|l|c|c|c|c|c|}
\hline Extent Score, Histologic Dx & Negative & Minimal & Moderate & Diffuse & Total \\
\hline Follicular Thyroid Adenoma & $41(85.42 \%)$ & $3(6.25 \%)$ & $3(6.25 \%)$ & $1(2.08 \%)$ & $\mathbf{4 8}$ \\
\hline Follicular Thyroid Carcinoma & $11(73.33 \%)$ & $1(6.67 \%)$ & $1(6.67 \%)$ & $2(13.33 \%)$ & 15 \\
\hline
\end{tabular}

being performed for benign thyroid nodules or morbidity associated with these operations. ${ }^{[2]}$ Some angiogenesis modulating genes such as VEGF have been suggested as a marker of more aggressive differentiated thyroid cancers ${ }^{[2]}$ or more strongly induced in differentiated malignant tumors in comparison with benign tumors. ${ }^{[16,27,28]}$

PSMA also known as folate hydrolase, is type II transmembrane glycoprotein that located on chromosome II. ${ }^{[15}$, ${ }^{29]}$ It was first described to be expressed in benign prostate acinar epithelium ${ }^{[15]}$ but further studies have shown that PSMA is also expressed in non-prostate tissue including luminal/apical aspect of duodenum, kidney proximal tubules and neuroendocrine cells in colonic crypt. ${ }^{[29]}$

Also, PSMA has been known as a tumor associated neovasculature marker in some solid malignant tumors ${ }^{[19]}$ which targeting of that by cytotoxic- conjugated antibody can represent powerful tool for vascular targeted therapy in malignant tumors. ${ }^{[15]}$ We did not find significant difference between extent of expression of PSMA between benign and malignant follicular neoplasms which limits its ability as a differentiating marker. In a study by Karl Sgal et al, ${ }^{[14,30]}$ a marginal difference was noted in the degree of vascularity between FTA and FTC using VWF by IHC method. An important finding of the study was increased density of vessels adjacent to capsule (especially in area of invasion) in FTC which was not the same in FTA(1 vessel/Two thyroid follicular cells versus 1 vessel /10 thyroid follicular cells, respectively). Also areas with solid proliferation showed increased vascularity. ${ }^{[14,30]}$ In another study by Frigugliett et al, ${ }^{[14,31]}$ using CD34 to estimate the angiogenesis, there was no significant difference between FTC and FTA $(p=0.388)$. Also there was no significance regarding to sex, size, or age. However, AcSDKP as an angiogenic active marker showed five times higher mean tissue concentration in PTC rather than benign nodular goiter by Kusinski et al. ${ }^{[18]}$ Working on the value of angiogenesis-modulating genes in various thyroid lesions by Kebebew et al,, ${ }^{[2]}$ some independent markers of malignancy of follicular cell origin were observed including ANGPT2 (Angiopeptin2)and TIMP1 (Tissue inhibitor of metalloproteinase I) mRNA. They recommended that they could be helpful adjunct to FNA cytology. ${ }^{[2]}$

Recently, there has been some evidences regarding to increased uptake of PSMA using $\left[{ }^{68} \mathrm{Ga}\right]$ PSMA-HBEDccPET/CT in different thyroid tumors. ${ }^{[22-26]}$ For example, in a report by Kanthan et $\mathrm{al}^{[23]}$ a known case of prostate cancer underwent $\left[{ }^{68} \mathrm{Ga}\right]$ PSMA-HBED-ccPET/CT for staging. A PSMA - Avid thyroid lesion was identified which on further histologic exam was diagnosed as FTA. ${ }^{[23]}$

Incidental FTC with increased uptake also reported by Sager et al, ${ }^{[24]}$ in a known case of prostate cancer. In another report by Tawade et $\mathrm{al}^{[25]}$ they believes that it was more effective in detection of multiple mediastinal, lung and supra-clavicular lymph nodes and brain metastatic lesions in a patient with rising thyroglobulin and negative whole body radioiodine scan. ${ }^{[25]}$

In a tissue microarray based study by Mhawech-Fauceglia $\mathrm{P}$ et $\mathrm{al}^{[32,33]}$ they observed positive PSMA staining in some isolated thyroid cancers. However no PSMA positivity was reported by their team in 846 benign tumors. ${ }^{[32,33]}$

On histology, However; there are limited data regarding to expression of PSMA in thyroid neoplasms. In a study by Silver et ${ }^{\left[{ }^{[29]}\right.}$ and Mhawech-Fauceglia et al., ${ }^{[33]}$ they found thyroid is negative for PSMA using clone mouse monoclonal antibody CYT-351(clone 7E11-C5;Cytogen, Princeton, NJ) and monoclonal antibody YPSMA1,1:50;Genetex,Inc., San Antonio, Tx, USA), respectively. We also did not find any reaction of thyroid follicular cell using monoclonal liquid NCL-L-PSMA mouse antibody (Clone 1D6, Novocastra). However regarding to tumor neovasculature, seven out of 48 and four out of 15 cases of FTA and FTC were positive for PSMA, respectively.

\section{Conclusion}

To our knowledge, this is the first study focusing on expression of PSMA in thyroid follicular neoplasms by IHC method. The intensity of PSMA in vascular structures of follicular thyroid carcinomas was significantly stronger than adenomas. However, Respect to thyroid follicular cell staining, our findings do not support usefulness of PSMA in differentiating between FTA and FTC. More importantly, expression of PSMA in neovasculature of differentiated thyroid follicular neoplasms can represent a potential utility for PSMA-targeted radionuclide therapy in advanced ${ }^{131} \mathrm{I}$-resistant/negative differentiated thyroid cancers.

\section{Acknowledgment}

The authors would like to thank the staff of Pathology and immunohistochemistry department for their devoted technical support. 


\section{Funding}

This research was funded by Tehran University of Medical Sciences as a residency thesis.

\section{References}

1. Wartofsky L. Increasing world incidence of thyroid cancer: Increased detection or higher radiation exposure?Hormones.2010;9(2): 103-108.

2. Kebebew E., Peng M, Reiff E, Duh Q, Clark O, McMillan A.Diagnostic and Prognostic value of angiogenesismodulating genes in malignant thyroid neoplasms. Surgery.2005; 138: 1102-10.

3. Duggal R, Rajwanshi A, Gupta N, Vasishta R. K.Interobserver variability amongst cytopathologists and histopathologists in the diagnosis of neoplastic follicular patterened lesions of thyroid". Diagn Cytopathol.2011;39(4): 235-241.

4. Sanii S., Saffar H, Moradi Tabriz H, Qorbani M, Haghpanah V, Tavangar S. M.Expression of Matrix Metalloproteinase-2, but not Caspase-3, Facilitates Distinction between Benigsn and malignant thyroid follicular neoplasm.Asian PacJ Cancer Prev.2012;13:

5. Yoon JH., Kim E.K, Youk J. H., Moon H. J., Kwak J. Y. Better understanding in the differentiation of thyroid folicular adenoma, follicular carcinoma, and follicular variant of papillary carcinoma: A retrospective study.Int J Endocrinol. 2014; ID 321595: (http://dx.doi.org/10.1155/2014/321595).

6. JehS.K., So L. J., Bum S. K., Yoen S. L. Evaluating the degree of conformity of papillary carcinoma and follicular carcinoma to the reported ultrasonographic findings of malignant thyroid tumor.Korean $\mathrm{J}$ Radiol. 2007;8(3): 192-197.

7. Jogai S., Adesina AO., Temmim L., Al-Jassar A., Amir T., Amanguno HG. Follicular variant of papillary thyroid carcinoma-a cytological study.Cytopathology. 2004;15(4): 212-216.

8. Vasko VV., Gaudart C, Allasia C. et al.Thyroid follicular adenomas may display features of follicular carcinoma and folicular variant of papillary carcinoma.Eur J Endocrinol.2004;151(6): 79-786.

9. Manivannan P. Siddaraju N., Jatia L., Kumar Verma S. Role of pro-angiogenic marker galectin-3 in follicular neoplasms of thyroid. Indian JBiochem. Biophys. 2012; 49: 392-4.

10. Dosen, D., Turic M. Smalcelj J., Gric MP., Separovic V. The value of frozen section in intraoperative surgical management of thyroid folicular carcinoma. Head Neck. 2003;25(7): 521-528.

11. Goldstein, R.E., Netterville J. L., Burkey B., Johnson J. E.Implications of Folicular neoplasms, atypia, and lesions suspicious for malignancy diagnosed by fine-needle aspiration of thyroid nodules. Ann Surg. 2002;235(5): 656-664.

12. McHenry C.R, Phitayakorn R. Follicular Adenoma and Carcinoma of the Thyroid Gland. Oncologist. 2011; 16: 585-593.
13. Finley DJ., Zhu B, Barden C. B., Thomas J. F. Discrimination of benign and malignant thyroid nodules by molecular profiling.Ann Surg. 2004;240(3): 425-437.

14. Sprindzuk MV. Angiogenesis in thyroid malignant neoplasm: State of the art and advances of the modern digital pathology and nanotechnology.J Clin Pathol Forensic Med.2010; 1(3): 16-34.

15. Wernicke A.G., Edgar M. A., Lavi E, Liu H, Salerno P, Bander N. H., Gutin P. H. Prostate-Specific Membrane Antigen as a potential novel vascular target for treatment of Glioblastoma Multiforme.Arch Pathol Lab Med.2011; 135: 1486-1489.

16. Itoh, A., Iwase K., Jimbo S., Yamamoto H., Kokubo M., Senda T., Nakai A., Nagagasaka T., Hibi Y., Seko T.Expression of vascular endothelial growth factor and presence of angiovascular cells in tissues from different thyroid disorders. World J Surg. $2010 ; 34(2): 242-8$.

17. Ishiwata T., Ino Y., Takei H., Oyama T., Morishita Y.Tumor angiogenesis as an independent prognostic indicator in human papillary thyroid carcinoma. Oncol Rep. 1998; 5: $1343-8$.

18. Kusinski M., Wdzieczak-Bakala J., Liu J.M., Bignon J., Kuzdak K.AcSDKP: a new potential marker of malignancy of the thyroid gland. Langenbecks Arch Surg. 2006;391: 9-12.

19. Chang, S.S., Reuter VE., Heston WD., Bander NH., Grauer LS., Gaudin PB.Five different Anti-Prostate-specific Membrane Antigen (PSMA) Antibodies confirm PSMA exprssion in Tumor-associated neovasculature. Cancer Res.1999;59: 3192.

20. Liu H, Moy P, Kim S, Xia Y, Rajasekaran A, Navarro V, Knudsen B, Bander N.H. Monoclonal antibodies to the extracellular domain of prostate-specific membrane antigen also react with tumor vascular endothelium.Cancer Res.1997; 57(17): 3629-3634.

21. Nomura N., Pastorino S., Jiang P., Lambert G., Crawford J.R., Gymnopoulos M., Piccioni D., Jaurez T., Pingle S.C., Makale M., Kesari S. Prostate_Specific Membrane Antigen expression in primary glioma and breast cancer breast metastasis.Cancer Cell Int. 2014;14(26).

22. Damle N.A., Tripathi M., Chakraborty P.S., Sahoo M. K., Bal C., Aggarwal S., Arora G., Kumar P., Kumar R., Gupta R.Unusual uptake of Prostate Specific Tracer 68GaPSMA-HBED-CC in a benign thyroid nodule. Nucl Med Mol Imaging. 2016; 50(4):344-347. DOI 10.1007/s13139016-0408-y.

23. Kanthan G.L., Drummond J., Schembri G. P., Izard M.A., Hsiao E. Follicular Thyroid adenoma showing Avid Uptake on 68Ga PSMA-HBED-CC PET/CT. Clin Nucl Med. 2016; 41(4): 331.

24. Sager S., Vatankulu B., Uslu L., Sonmezoglu K. Incidental detection of follicular thyroid carcinoma in 68Ga-PSMA PET/CT imaging. JNMedTechnol.2016; 44: 199-200. 
25. Taywade S.K., DamleN.A., and BalC.PSMA expression in papillary thyroid carcinoma. Opening a new horizon in management of thyroid cancer? Clin Nucl Med. 2016;41(5).

26. Verburg F.A., Krohn T., Heinzel A., Mottaghy F. M., Behrendt F. F.First evidence of PSMA expression in differentiated thyroid cancer using [68Ga]PSMA-HBED-CC PET/CT. Eur JNucl Med Mol imaging.2015; 42: 1622-1623.

27. Fenton C., Patel A., Dinauer C., Robie D. K., Tuttle R. M., Francis G. L.The expression of vascular endothelial growth factor and the type 1 vascular endothelial growth factor receptor correlate with the size of papillary thyroid carcinoma in children and young adults. Thyroid.2009;10(4): 349-57.

28. Nadoushan M.J., Siadati S., Amin A. A., Davati A., Mirzapour M. Study ofcorrelation between vascular endithelial groeth factor receptor and some prognostic factors in papillary carcinoma of thyroid gland. J Basic Clinpathophysiol. 2012;1(1): 1-7.

29. Silver D A., Pellicer I., Fair WR., Heston W. D., CordonCardo C . Prostate-Specific membrane antigen expression in normal and malignant human tissues.Clinical cancer Res.1997;3: 81-85.
30. Segal K, Shpitzer T, Feinmesser M, Stern Y, Feinmesser R. Angiogenesis in follicular tumors of the thyroid. JSurg Oncol. 1996; 63:95-98

31. Frigugliett IC, Mello ES, Castro IV, Filho GB, Alves VA .Metalloproteinase -9 immunoexpression and angiogenesis in thyroid follicular neoplasms: relation to clinical and histopathologic features. Head Neck.2001; 22(4):373-382.

32. Chaux A., Eifler J., Karram S., Al-Hussain T., Faraj S., Pomper M., Rodriguez R., George J. Netto G.Focal Positive Prostate-specific membrane antigen( SMA) expression in ganglionic tissues associated with prostate neovasculature bundle: Implication for novel intraoperative PSMA-based fluorescent imaging techniques.Urol Oncol.2011;31(5): $572-575$.

33. Mhawech-Fauceglia P, Zhang S, Terracciano L, Sauter G, Chadhuri A, Herrmann F R, PenetranteR.Prostate-Specific Membrane antigen(PSMA) protein expression in normal and neoplastic tissues and its sensitivity and specificity in prostate adenocarcinoma: an immunohistochemical study using multiple tumor tissue microarray technique. Histopathology. 2007;50: 472-483.

*Corresponding author:

Hiva Saffar, Associate Professor of Anatomical and Clinical Pathology, Department of Pathology, Shariati Hospital, Tehran University of Medical Sciences, Tehran, Iran.

Phone: +91 982184902187

Email: hsaffar@sina.tums.ac.ir

Date of Submission : 06.05.2017

Financial or other Competing Interests: None. 